\title{
The Development Status and Market Analysis of Human- computer Interaction
}

Jiayi Hu 1, a, Qianchen Ma 2, b, Sizhou Liu 3, c, Zewei Liu 4, d, Zirui Luo 5, e, Yaxin Li ${ }^{6, f}$, Chuanpu Liu ${ }^{7, g}$, Kunxuan Lu ${ }^{8, h}$, Hanxia Li ${ }^{9, i}$

1. School of Mathematics, Sichuan University, Chengdu 610065, China

2. College of Art and Science, New York University, 10003, United States

3. School of explore, Purdue University, 47907, United States

4. Lasell University, Boston, United States

5. Weston High School, 02493, United States

6. Surrey International Institute, Dongbei University of Finance and Economics, Dalian 116000, China

7. Saint Louis University, Saint Louis University (SLU) 1818 Advanced College Credit Program, 63103 United States

8. Chapel Hill- Chauncy Hall, 785 beaver St, Waltham, Massachusetts, 02452 United States

9. Tandon School of Engineering, New York University, 11201, United States

a jiayihu.com@outlook.com, b ${ }^{b}$ m335@nyu.edu, cliu3365@purdue.edu, dzeliu@lasell.edu, e22luoz@weston.org, ${ }^{\dagger}$ liyaxinelaine@163.com, 9 pekchuanpu@icloud.com, h916089094@qq.com, ihl3962@nyu.edu

Abstract. Human-computer interaction is one of the main branches of computer science, which aims to study how computers, robots and other systems interact with users in a reasonable manner. In this paper, we reviewed the development history and current situation of Human-computer interaction. And, the current application of Human-computer interaction in vertical industry is introduced. Finally, we analyze the market development prospect of Human-computer interaction in the future.

Keywords: Human-computer Interaction; Market Analysis; Perception Technology.

\section{Introduction}

The concept of Human-computer Interaction (HCI) advented in the 20th century. Its development can be summed up to a process from humans adapting machines to machines adapting humans. The first phase of HCI was the usage of Electronic Numerical Integrator and Computer (ENIAC) in World War two for calculating artillery firing tables with large portions of numbers by putting order into the machine artificially, which, however, takes days to accomplish. [1] More advanced HCI is humans manipulated machines by coding, keys, mouses within a Graphical User Interface (UI), one representation is the Windows system. After which is the latest development of HCI which contains Conversation Analysis (CA), applications on Virtual Reality (VA), and so on. More advanced HCI involves voice input from humans which not only requires machines to understand user's speeches by extracting the voice input from the wavelength and later transfer it to signals which can be analyzed within a large dataset but also require the skill of Conversation Analysis. [2 CA provides machines the ability to understand the voice input beyond simple commands such as setting an alarm or opening certain apps [3]. atest development of HCI also contains gesture analysis, which focuses on human motion monitor and facial gesture analysis. Monitoring of the lips gesture helps correct the error in voice analysis from the user. While eye tracking can monitor the eyesight of the user. These developments are beyond the scope of traditional HCI which focuses on simply giving commands. 
The latest research on HCI focuses on multimodality instead of unimodality. This means that the input from the user is not just simply one facet by the combination of them all. And machines use these analyses to calculate the status of the human which can make a more accurate prediction. HCI has covered multi-dimensional research areas, not just Computer Science, but also psychology, cognitive science, and even Medicine. The ultimate goal for HCI now is Ubiquitous computing, or "everyware", where computing is anytime and anywhere, even without being constrained by the existence of a computer [4].

Human-computer interaction (HCI) or also known as human-machine interaction (HMI) is a field of research that focused on interfaces between the computer and its users. HCI is a field that involves multiple disciplines that including computer science such as programming language, software programming and also human science for example cognitive psychology [5]. The first known use of term HCI was in 1975 and became popular in the Stuart K. Card, Allen Newell, and Thomas Moran's book that published in 1983 named that "The Psychology of Human-computer Interaction." In order to improve the interaction between the human and the computer, the majority of research about HCI in the past concerned the design, implementation and assessment of new interfaces. The Humancomputer interface can be divided into input and output of information which have visual base and Audio base. For now, the visual based interaction is the most widespread area in Human-computer interaction research [6].

Nowadays, the keyboard-mouse paradigm also known as WIMP (windows, icons, menus, pointand-click devices) is still the most popular around the world. In the future research, the HCI researchers will spend more resources and pay more attention on the usability of software and hardware of computer. According to the international standards organization, the usability of HCI means the effectiveness, efficiency and satisfaction [7].

Human-Machine Interaction(HMI) was designed that humans would be interacting with the machine automatically. It played a huge role in our daily life. For example, a keyboard, a mouse, an ipad, are all HMI. It also played a huge role in industry and manufacturing. The top 3 of the vendors right now are IPCS Automation, an American multinational company, Livewire, an IT training institute, and Mitsubishi Electric, a Japanese company which made automation systems and provided it to different fields. These three companies are the examples of this growing market. According to the report from the Grand View Research, the market of HMI might be worth $\$ 6.61$ billion by 2022 [8]. People now had seen the advantages and benefits from the use of HMI to the treatment of oil and water, so they "instigated companies to develop devices with better wear and tear properties", and that's one of the reasons why the market was predicted to grow. However, because of the competitive relationship between each company, they were more focused on the development of systems that targeted specific problems, which could help them earn the advantage in the competition. The demand for more advanced panels instead of the traditional one was also needed, and that could be a part of the future development [9]. Moreover, HMI products that were used in daily life also need improvement. For instance, a wearable device would be popular for the consumers. People would be more likely to use and buy more convenient products. Lastly, the market would have a huge growth if the HMI companies could develop systems for the manufacturers to help them improve their productivity [10].

\section{Perception Technology of Human-computer Interaction and Industrial Development}

Human machine interaction technology relates to many aspects of human biological features and the cooperation of machines. Three popular recognition technologies are introduced here. They are speech recognition, hand gesture recognition, and face recognition, respectively.

Speech recognition is defined to be the process of converting voice signals to word sequences. People's voice conveys lots of information, such as gender, age, mood, status, etc. The purpose of speech recognition is to determine the identification of the speaker through characteristics of the 
speaker's voice. A speech can be divided into isolated word system, connected word system, continuous speech system, and spontaneous speech system [11]. Speech recognition extracts and matches the features of the speaker to create speaker modeling. Principal Component Analysis (PCA), Linear Discriminate Analysis (LDA) and Mel-Frequency Cepstrum (MFCCs) are common methods to extract features. In feature matching process, various techniques like Dynamic Time Warping (DTW), Vector Quantization (VQ), Linde-Buzo-Gray (LBG) Algorithm are widely used and each of them has its specification [12].

Hand gesture recognition is a natural way of human machine interaction. The objective of hand gesture recognition is to build a system that conveys information or control equipment by recognizing specific hand gestures. The components of hand gesture system include separating hands from the background, tracking hands, extracting features of hands through multiple algorithms, classifying and postprocessing gestures. Commonly used learning algorithms, such as neural networks, instancebased learning, and hidden Markov models, stem from artificial intelligence. Hand gesture recognition is broadly applied to many fields: monitoring patients medically, video conferencing, and graphic editor control, etc. [13].

Face recognition is another significant part in human machine interaction. It has two main objectives--one is verification, and the other is identification. Major human face recognition techniques include eigenfaces, neural networks, dynamic link architecture, graph matching, and 3D morphable model. They focus on analyzing facial expressions. In the process, biometric devices complete three steps. First, face observation, which indicates the signature of the subject. Second, sample standardization. It coordinates observations to make them have the same formations as those in data sets. Finally, comparison. A matcher compares them to the data stored in the computer and we get the analysis on the similarity between observations and stored data. Face recognition is highly valued in health care, security and defense, retail and marketing [14].

\section{The Application of Human-computer Interaction and its Market Analysis}

\subsection{Typical Applications of HCI}

Application in medical field

Computer-assisted surgery (CAS) is a wide field of technologies with the potential to enable healthcare providers to improve efficiency and efficacy of diagnosis, treatment, and clinical management. There is no single definition of CAS, and definitions vary widely since many different applications for computer assistance in surgery exist. An example of CAS is robot-assisted surgery. In recent years, with the rapid development of the medical industry, computer-based virtual reality technology, which is a part of the Human-computer interaction technology, has penetrated various fields of medicine [15]. Young surgeons need a lot of detailed training and practice before they walk to the operating table, and a virtual reality (VR) can help building a computer-generated environment that uses visual, auditory, and tactile effects on the user to create an immersive interactive visual simulation. $80 \%$ of the surgical errors are caused by human factors, the VR practice shows its importance in increasing surgical success rate and saving more lives [16].

VR has also been used in the treatment of anxiety disorders and addiction disorder. Virtual reality cognitive behavioral therapy (CBT) for substance dependence combines traditional CBT/relapse prevention training with exposure and skill practice in VR-based environments (e.g., bar, party, smoking lounge in an airport). Virtual reality CBT allows patient to acquire and practice skills in an environment that closely resembles the real world setting in which they will be performed. These methods have been successfully employed for treatment of fear of flying; social phobia; claustrophobia; post-traumatic stress disorder in soldiers and in World Trade Center workers and rescuers; and attention deficit hyperactivity disorder; and for stroke rehabilitation [17].

Application in gaming

In fact, Human-computer interaction system and video games are consistent. The use of gesture interaction system can replace the use of mouse and give players a much better experience, the use 
of VR can change the game mode to enhance the sense of participation, the use of intelligent voice can increase the interaction between players and the game. The development of future games must be inseparable from Human-computer interaction.

Application in industry

In industrial field, human-robot collaboration is a popular topic. For example, the research project "Collaborative Robotics" (CollRob), is an initiative focusing on the conceptualization, research, development, and evaluation of novel methods and tools for collaborative and cooperative robots. With the robot equipped with torque sensors, people can use a tablet, augmented reality (AR) glasses, microphones, speakers, and gesture bracelets to control the robot. The safety of controllers is also guaranteed by bio-sensors and eye tracking glasses worn by them.

Application in agriculture

The gesture interaction system, a new Human-computer interactive way, is going to play a huge role in both the virtual agricultural field and traditional agricultural field. In the virtual agricultural field, objects are manipulated in virtual environment by gesture, people can accurately simulate the plant shape of crops to achieve the maximum yield, and intuitively simulate the row spacing of intercropping and accompanying crops, providing a useful reference for the rational planning of cultivated land. In traditional agricultural field, gesture recognition interaction will reduce the barriers to the use of computers and help farmers use advanced systems for agricultural production. It is also more convenient to carry a gesture interaction machine rather than a computer, which makes remote control easier for farmers.

\subsection{Market Analysis of Human-computer}

For an overview of the HCI application market, a PEST analysis was adopted. Below are the results.

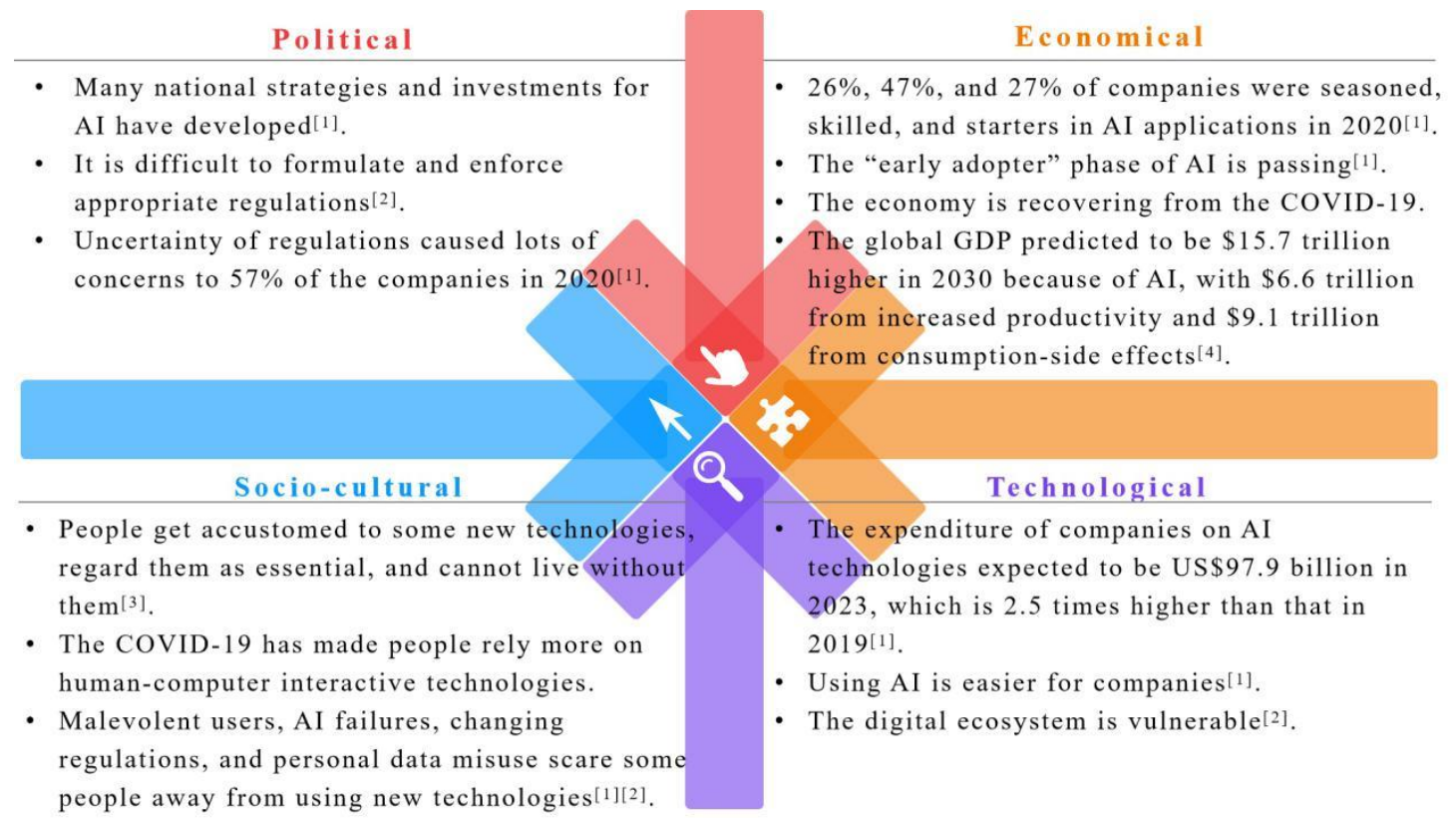

Figure 1. Results of PEST Analysis

In the political aspect, it is clear that governments are paying great attention to the development of Artificial Intellectual (AI) technologies like HCI. Many national strategies and investments for AI have developed[18]. For example, there were 15,700 investments for AI start-ups during 2014 and 2019[19]. However, due to unanimous norms worldwide, it is difficult to formulate and enforce appropriate regulations[20]. Governments are eager to become advanced in new technologies and are too worried to make regulations due to the possible negative impacts. Nevertheless, this uncertainty of regulations caused lots of concerns to $57 \%$ of the companies adopting AI in 2020 and may hinder the advancing process. 
Economically, the applications of AI technologies will make many contributions to the world economy. As is shown in the investigation of Deloitte, $26 \%, 47 \%$, and $27 \%$ of companies were seasoned, skilled, and starters in AI applications in 2020. As more and more companies adopt the technologies, the "early adopter" phase of AI is passing. Although the COVID-19 was a great shock, the economy is recovering now. The global GDP predicted to be $\$ 15.7$ trillion higher in 2030 because of AI, with $\$ 6.6$ trillion from increased productivity and $\$ 9.1$ trillion from consumption-side effects [21].

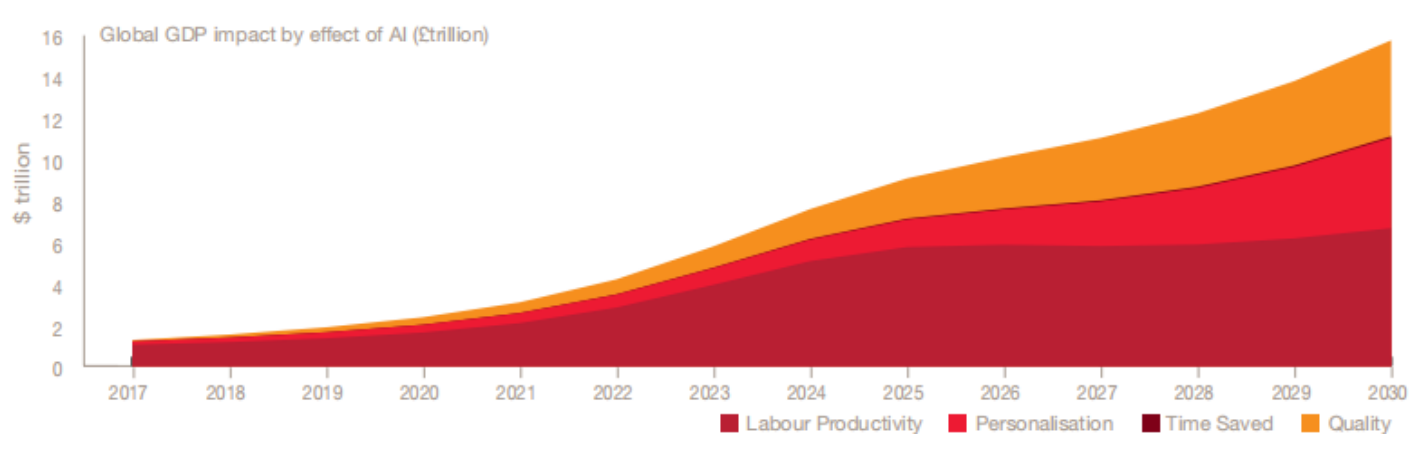

Figure 2. Where will the value gains come from with AI?

Thirdly, some sociocultural factors may influence the application of HCI. First, the living style and value of people are changing. People get accustomed to some new technologies, regard them as essential, and cannot live without them. Moreover, the pandemic COVID-19 has made people rely even more on HCI technologies. Nonetheless, malevolent users, AI failures, changing regulations, and personal data misuse scare some people away from using new technologies. As for technological factors, technologies are growing very fast since every country wants to get advantages in the future, though some problems need to be solved. The expenditure of companies on AI technologies expected to be $\$ 97.9$ billion in 2023 , which is 2.5 times higher than that in 2019 With the rapid developments, using AI is easier for companies As the digital ecosystem is vulnerable, there should be some concerns. Overall, the macro-environment for HCI application businesses is pretty good. For better evolvement, there should be clearer regulations and better protection for cybersecurity [22].

\section{Conclusion}

Human-computer interaction technology is an important branch of computer science. From the analysis of the development mileage of Human-computer interaction, Human-computer intelligent interaction makes it more convenient for people to operate computers, and promotes computers to enter all aspects of people's lives, becoming an essential daily tool. This article starts with a typical Human-computer interaction application, introduces the application of Human-computer interaction in vertical industries, and analyzes its future market size.

\section{References}

[1] Erdel T, Crooks S. Speech recognition technology: an outlook for human-tomachine interaction[J]. Journal of healthcare information management: JHIM, 2000,14(2):13-21.

[2] Kantlavania K , Sharma S , Sharma K K . Reviewing Human-Machine Interaction through Speech Recognition approaches and Analyzing an approach for Designing an Efficient System[J]. International Journal of Computer Applications, 2012, 38(3):26-32.

[3] Juang B H , Furui S . Automatic recognition and understanding of spoken language - a first step toward natural human-machine communication[J]. Proceedings of the IEEE, 2000, 88(8):1142-1165.

[4] Hasan H S , Kareem S . Human Computer Interaction for Vision Based Hand Gesture Recognition: A Survey [J]. Artificial Intelligence Review, 2013, 43(1):1-54. 
[5] Smith A, Sutherland A I , Lemoine A, et al. Hand gesture recognition system and method[J]. US, 2000.

[6] Xu P . A Real-time Hand Gesture Recognition and Human-computer Interaction System. 2017.

[7] Tistarelli M , Barrett S E, O'Toole A J . Facial Recognition, Facial Expression and Intention Detection[M]. Springer Netherlands, 2012.

[8] Brunelli R , Poggio T . Face recognition: features versus templates[J]. IEEE Trans.pattern Anal. \& Mach.intell, 1993, 15(10):1042-1052.

[9] Tanaka J W , Farah M J . Parts and wholes in face recognition.[J]. Quarterly Journal of Experimental Psychology A, 1993, 46(2):225-245.

[10] Huang, Z., Bo, P., \& Wu, J. (2012). Research and Application of Human-computer Interaction System Based on Gesture Recognition Technology. International Conference on Computer \& Computing Technologies in Agriculture. Springer Berlin Heidelberg.

[11] Liu, S. , \& Li, T. . (2020). Application of Human-computer interaction technology in laparoscopic surgery. IOP Conference Series Materials Science and Engineering, 750, 012129.

[12] Kenngott, H. G, Wagner, M., Nickel, F., Wekerle, A. L., Preukschas, A., \& Apitz, M., et al. (2015). Computer-assisted abdominal surgery: new technologies. Langenbeckl"s Archives of Surgery, 400(3), 273-281.

[13] Bordnick, P. S., Carter, B. L., \& Traylor, A. C. (2011). Virtual reality technologies for research and education in obesity and diabetes - sponsored by the national institutes of health and department of defense: what virtual reality research in addictions can tell us about the future of obesity assessment and t. Journal of Diabetes Science \& Technology, 5(2), 265-71.

[14] Velik, R., Dieber, B., Yahyanejad, S., Brandsttter, M., \& Hofbaur, M. (2016). A Step Forward in HumanRobot Collaboration --The Project CollRob. 1st OAGM \& ARW Joint Workshop on "Computer Vision and Robotics".

[15] Ammanath, B., Hupfer, S., \& Jarvis, D. Thriving in the era of pervasive AI[EB/OL].2020[2021-04-01].

[16] Gaines, B. R. From facilitating interactivity to managing hyperconnectivity: 50 years of human-computer studies [J]. International Journal of Human-computer Studies, 2019, 131:4-22.

[17] Ling, R. S. Taken for Grantedness: The Embedding of Mobile Communication into Society[M]. 2012.

[18] Rao, A., \& Verweij, G. Sizing the price: What's the real value of AI for your business and how can you capitalize? [EB/OL].PwC.2017[2021-04-01].

[19] Ammanath, B., Hupfer, S., \& Jarvis, D. (2020). Thriving in the era of pervasive AI. Deloitte Development LLC.

[20] Gaines, B. R. (2019). From facilitating interactivity to managing hyperconnectivity: 50 years of humancomputer studies. International Journal of Human-computer Studies, 131, 4-22. https:// doi. org/ 10. 1016 /j. ijhcs.2019.05.007.

[21] Ling, R. S. (2012). Taken for Grantedness: The embedding of Mobile Communication into Society. MIT Press.

[22] Rao, A., \& Verweij, G. (2017). Sizing the price: What's the real value of AI for your business and how can you capitalise? PwC. 